\title{
Direct Measurement of Atom Diffusion Using Atom-Tracking STM
}

\author{
B. S. Swartzentruber, R. Stumpf, T. R. Mattsson, and P. J. Feibelman
}

Sandia National Laboratories, PO Box 5800, Albuquerque, NM 87185-1415

It is a long-standing course of study to investigate the dynamic behavior of atoms and molecules at surfaces and interfaces in order to understand diffusion, reaction rates, growth, and alloying. This has become even more important with the recent push to develop ever smaller functional electronic and mechanical structures, in which, at the lowest limit, a few atoms out of place can destroy their utility. Understanding the details of the transition states is necessary to uncover the principles that govern surface chemistry. In addition, knowledge of the nature of available reaction paths allows us to systematically modify the kinetics of an atomic system and thus to modify its time evolution and structure. In this paper we present detailed measurements of the diffusion of adsorbed Si dimers on the $\mathrm{Si}(001)$ surface (Fig. 1) using conventional and atom-tracking scanning tunneling microscopy (STM). We controllably alter the diffusion rate using the electric field between the STM probe tip and the surface. The field dependence coupled with first-principles density functional theory (DFT) calculations of transition state electronic structure yields valuable insight into the elusive transition state.

In atom-tracking mode the STM probe tip is locked onto a selected dimer using lateral feedback [1]. Once locked, the feedback electronics maintain the tip over the cluster as it diffuses over the substrate tracking its coordinates (Fig. 2). Because the instrument is spending all of its time measuring the kinetics of the diffusing dimer, the time resolution is increased by more than 1000 over conventional STM imaging techniques. By measuring the diffusion statistics of the dimers as they meander near surface steps and defects we can extract subtle binding energy differences from lattice site to lattice site due to the dimer-defect interactions.

Because the tip is continually maintained over the position of the adsorbed dimer during atom tracking, the dimer is constantly exposed to the electric field between the tip and the sample. This advantageously enables a comprehensive measurement of how the electric field alters the motion of the dimer [2].

As shown in Fig. 3, our DFT calculations of the electric field dependence of diffusion show that the generally accepted (lowest-barrier) diffusion mechanism, so-called "piecewise diffusion", has the opposite field dependence to what is observed and therefore cannot be active. Furthermore, we discovered a different diffusion path, "walking" diffusion, with a similarly low barrier that has the correct field dependence. In walking diffusion the atoms of the adsorbed dimer remain bonded during the entire path, rotating as they pass through the transition state, as opposed to piecewise diffusion in which the atoms are separated at the transition state.

By increasing the capability of the STM to yield quantitative measurements that were previously inaccessible, we provide additional insight into the complicated interplay between processes at work during growth and etching. We can also identify and measure new dynamic mechanisms that exist on the surface. The quantitative measure of individual atomic-scale energy parameters not only 
serve as inputs to realistic simulations and model calculations, but also enable the validation and refinement of such calculations [3].

[1] B. S. Swartzentruber, Phys. Rev. Lett. 76, 459 (1996).

[2] J. M. Carpinelli and B. S. Swartzentruber, Phys. Rev. B58, 13423 (1998).

[3] Sandia is a multiprogram laboratory operated by Sandia Corporation, a Lockheed Martin Company, for the United States Department of Energy under contract DE-AC04-94AL85000. This work was supported in part by the Division of Materials Science and Engineering, Office of Science, U.S. Department of Energy.
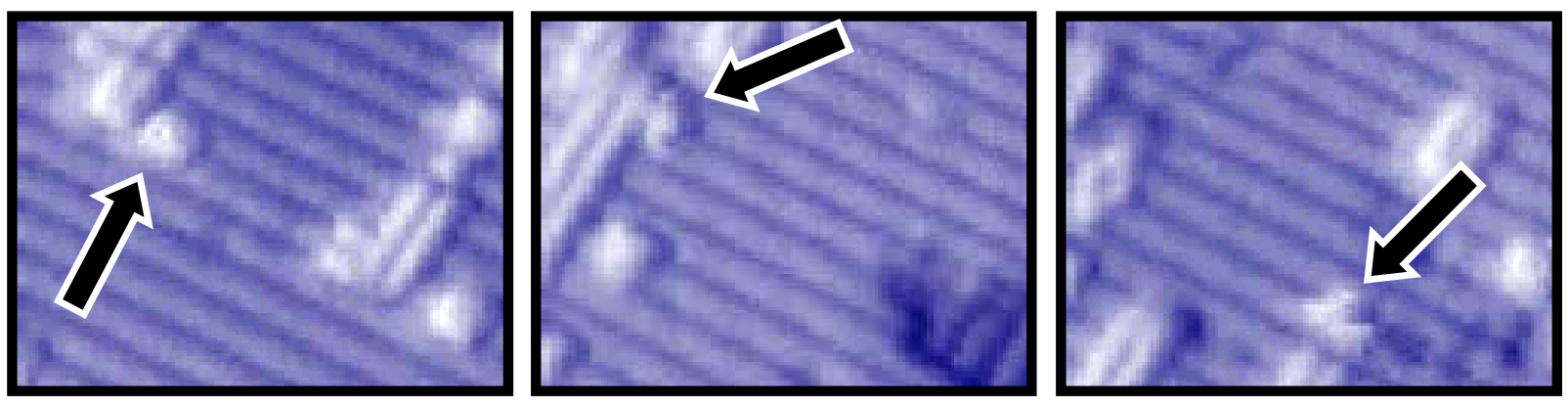

FIG 1: 100§ STM images of diffusing Si dimers (arrows) trapped between surface defects acquired at $\sim 100 \mathrm{C}$.

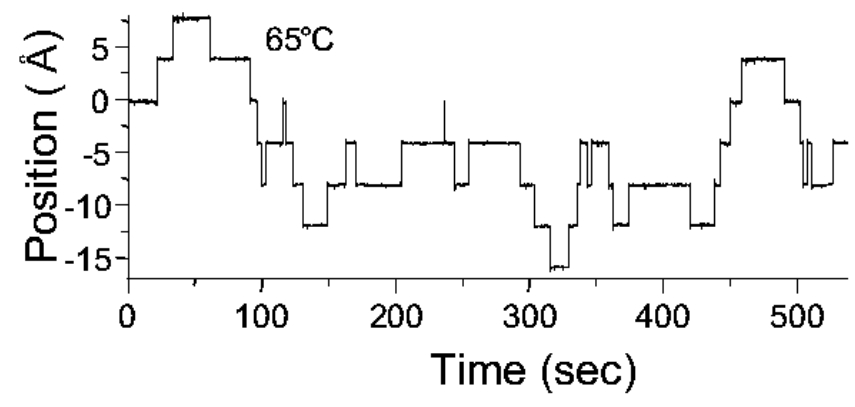

FIG 2: Plot of the position of a diffusing $\mathrm{Si}$ dimer as a function of time acquired at $65 \mathrm{C}$. The atom tracker resolves every diffusion event. The statistics of the diffusion process can be measured explicitly.

FIG 3: Si dimer diffusion barrier as a function of electric field relative to the zero-field value. Circles are the measured data. DFT values for "piecewise" diffusion (gray) and "walking" diffusion (black) are shown as lines - dashed lines are for LDA and solid lines are for GGA. Walking diffusion is the dominant transport mechanism.

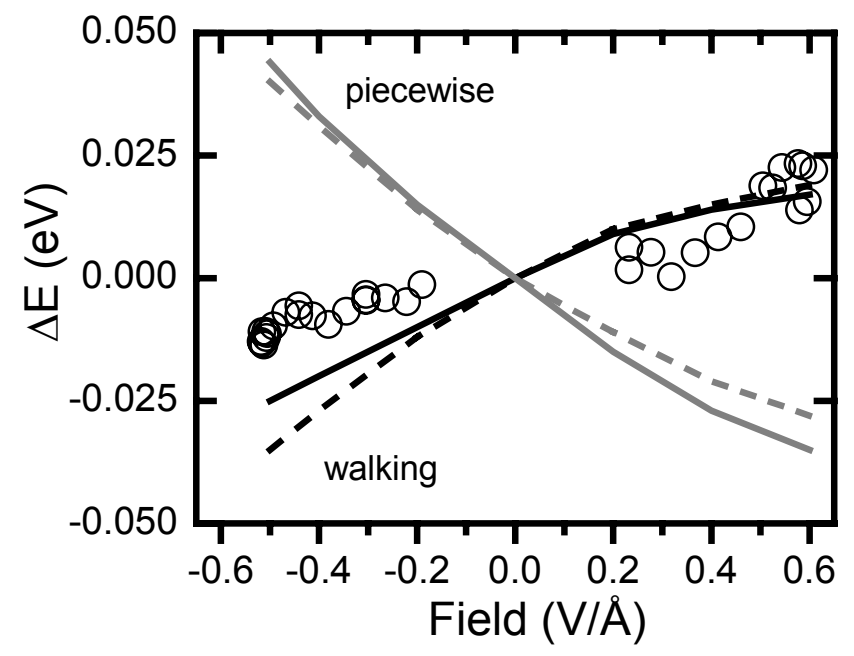

